\title{
Lipödem - Mythen und Fakten Teil 2
}

T. Bertsch'; G. Erbacher ${ }^{1,2}$

${ }^{1}$ Földiklinik Hinterzarten - Europäisches Zentrum für Lymphologie; ${ }^{2}$ Dipl.-Psychologin, Psychologische Psychotherapeutin, Supervisorin (hsi)

\section{Schlüsselwörter}

Lipödem, Ödem, Manuelle Lymphdrainage, Wissenschaftliche Evidenz

\section{Zusammenfassung}

In dieser kleinen Übersichtsreihe über die Mythen des Lipödems werfen wir einen kritischen Blick auf populäre Statements zum Lipödem; Statements, die vor Jahrzehnten schon Eingang in wissenschaftliche Publikationen gefunden haben und seither unkritisch und stetig wiederholt werden; Statements, die dadurch inzwischen zum selbstverständlichen Wissensallgemeingut von Lipödempatientinnen und vor allem auch von Lipödem-Selbsthilfegruppen geworden sind. Im ersten Teil unserer Darstellung haben wir uns kritisch mit zwei populären Mythen über das Lipödem auseinandergesetzt. Hierbei haben wir festgestellt, dass sowohl für das Statement "Das Lipödem ist eine progrediente Erkrankung" als auch für das Statement "Ein Lipödem macht psychisch krank" keine wissenschaftliche Evidenz vorliegt. In diesem zweiten Beitrag über die Mythen des Lipödems fokussieren wir uns auf den Ödemaspekt, auf das „Ödem im Lipödem" und die hieraus erfolgte therapeutische Konsequenz - die Manuelle Lymphdrainage. Daher: Mythos 3: Das Lipödem ist in erster Linie ein "Ödem-Problem"; daher ist die Manuelle Lymphdrainage essenzielle und regelmäßig durchzuführende Standardtherapie! Auch dieses Statement widerspricht in hohem MaBe unserer seit Jahren bestehenden täglichen klinischen Erfahrung mit diesem speziellen Patientengut. Gleichzeitig haben wir im Rahmen unserer umfangreichen Literaturrecherche festgestellt, dass es keine Evidenz für diese Sichtweise gibt. Tatsächlich gibt es keinerlei Hinweis darauf, dass beim Lipödem ein relevantes Ödem - Ödem im Sinne von Flüssigkeit - vorliegt. Ebenso fehlt jegliche wissenschaftliche Evidenz dafür, dass dieses kaum (bzw. meist nicht) vorhandene Ödem für die Beschwerden der Lipödempatientinnen verantwortlich ist. Der regelmäßigen und dauerhaften Verordnung von Manuellen Lymphdrainagen mit dem Ziel der "Ödembeseitigung" fehlt daher jede Grundlage. Das Lipödem ist weit mehr als nur dickere, schmerzhafte Beine! Darum müssen wir manche der alten therapeutischen Pfade verlassen, Pfade, für die es keine wissenschaftliche Evidenz gibt, Pfade, die darüber hinaus auch unserer klinischen Erfahrung widersprechen. Eine umfassende Therapie des Lipödems sollte daher auch all jene Aspekte berücksichtigen, die nicht so offensichtlich sind wie das Augenscheinliche und das vordergründig Geäußerte. Lipödem-Therapie muss neben der Behandlung der somatischen Beschwerden auch auf die bereits in unserem ersten Beitrag beschriebenen psychosozialen und gesellschaftlichen Aspekte dieses komplexen Krankheitsbildes fokussieren. Die Vorstellung eines umfassenden Therapiekonzeptes für Lipödempatientinnen wird Inhalt im letzten Teil unserer kleinen Lipödemreihe sein. Neue Wege entstehen, in dem wir sie gehen dies gilt auch für die Therapie des Lipödems!

Lipoedema - myths and facts Part 2

Phlebologie 2018; 47: 120-126

https://doi.org/10.12687/phleb2418-3-2018

Eingereicht: 12. März 2018

Angenommen: 12. März 2018

English version available at:

www.thieme.de/phlebo

\section{Keywords}

Lipedema, edema, manual lymph drainage, scientific evidence

\section{Summary}

Lipedema as a disease is associated with numerous myths. In this small overview of the myths surrounding lipedema, we throw a critical eye on popular statements regarding the disease; We have found that statements made in scientific publications decades ago have been repeated over and over again without criticism. These statements have become part of the general knowledge for lipedema patients and lipedema self-help groups. In the first part of our presentation we focussed on critically reviewing two popular myths about lipedema. We found that there were no scientific evidence for the following statements: "Lipedema is a progressive disease", and "Lipedema negatively affects mental health". In this our second contribution on the myths surrounding lipedema, we focussed on the edema aspect; i.e. on the so-called "edema in lipedema" and the subsequently recommended therapy - manual lymph drainage. Myth \#3: Lipedema is primarily an "edema problem", and manual lymph drainage is thus an essential standard form of therapy, which must be conducted regularly! This statement also contradicts our daily experiences with this specific subset of patients to a high degree. Simultaneously we also established through extensive literature research, that there is no evidence for this concept. There is actually no indication that any form of relevant edema is present in lipedema patients, i.e. edema in the sense of fluid retention. There is also no scientific evidence that this barely measureable (in most cases entirely absent) edema is reponsible for the complaints of lipedema patients. There is thus no basis for the prescription of long-term and regular manual lymph drainage for treating this "edema". Lipedema is much more than just fat and pain- 
ful legs! We must thus leave behind some of the old therapeutic methods, for which there is no scientific evidence and which furthermore directly contradict our clinical experiences. The comprehensive treatment of lipedema should thus consider all aspects of the disease, not only the immediately obvious such as observable changes and reported symptoms. Lipedema therapy must focus on the treatment of somatic complaints as well as on the psychosocial and sociological aspects of this complex disease, as outlined in our first contribution. The presentation of a comprehensive therapeutic concept for lipedema patients will be the subject of the last part of our short series on lipedema. We find new paths when we have the courage to walk down them - this is also true for the treatment of lipedema!

\section{Einleitung}

Das Lipödem ist weitaus mehr als dickere, schmerzhafte Beine! Allerdings - auch das sei betont - nicht jedes dickere Bein ist ein Lipödem!

In der Földiklinik in Hinterzarten - Europäisches Zentrum für Lymphologie - behandeln wir jedes Jahr ca. 3000 Patientinnen mit der Diagnose Lipödem - ambulant und stationär. Die überwältigende Mehrheit dieser Patientinnen kommt mit Vorstellungen und Erwartungen in unsere Klinik, die durch Medien, aber auch durch Ärzte genährt wurden; Vorstellungen über das Lipödem, die weit entfernt sind von wissenschaftlicher Evidenz; Erwartungen, die oft jenseits des Machbaren liegen.

Über das Lipödem kursieren zahlreiche Mythen; Statements, die Eingang in die Wissenschaft und damit auch Eingang ins „Patienten-Know-how" gefunden haben. Im ersten Teil unserer kleinen Reihe über das Lipödem haben wir bereits zwei dieser Mythen zum Lipödem diskutiert (1):

- Mythos 1: Das Lipödem ist eine chronische, progrediente Erkrankung

- Mythos 2: Ein Lipödem macht psychisch krank

In diesem Beitrag über die Mythen des Lipödems fokussieren wir uns auf den Ödem- aspekt, auf das „Ödem im Lipödem“ und die hieraus erfolgte therapeutische Konsequenz, die Manuelle Lymphdrainage.

- Mythos 3: Das Lipödem ist in erster Linie ein „Ödem-Problem“; daher ist die Manuelle Lymphdrainage eine essenzielle und regelmäßig durchzuführende Standardtherapie!

Dieses Statement scheint ein „lymphologisches Dogma“ zu sein. In einer Vielzahl von nationalen als auch internationalen Publikationen wird ebenso wie in Lipödemportalen im Internet die Manuelle Lymphdrainage als essenzielle Standardtherapie des Lipödems betrachtet (2-5). Herpertz schlägt eine „ambulante Behandlungsfrequenz beim Lipödem in der Regel mit 1- bis 2-mal wöchentlich als 45- oder in schweren Fällen als 60-Minuten-Behandlung" vor (6). Ein von Lipödempatientinnen häufig frequentiertes Internetportal schreibt: Patientinnen mit Lipödem „,benötigen in aller Regel eine dauerhafte Behandlung mit Manueller Lymphdrainage“ (7).

Hintergrund dieser übereinstimmenden Therapieempfehlung ist die feste Überzeugung, dass das Lipödem in erster Linie ein „Ödem-Problem“ sei, dieses Ödem daher auch primär behandelt werden müsse. Meier-Vollrath et al. schreiben im Deutschen Ärzteblatt: „Ziel der Behandlung ist die Ödembeseitigung“ (8).

Diesem Dogma liegt eine Sichtweise auf das Lipödem zugrunde, die ihren Ursprung in der aus heutiger Sicht unglücklichen Namensgebung dieser Erkrankung durch die Erstbeschreiber E.V. Allen und E.H. Hines im Jahre 1940 hat. Bei genauer Betrachtung der Originalliteratur von 1940 und 1951 drängt sich allerdings der Eindruck auf, dass diese in wesentlichen Teilen fehlinterpretiert wurde. So spielt das „Ödem" in beiden Artikeln eine eher untergeordnete Rolle. In der Erstpublikation wird ausführlich die Pathophysiologie des orthostatischen Ödems erklärt, um dann $\mathrm{zu}$ folgern: „This is the situation in cases of lipedema“, und weiter: „Near the end of a day of activity some edema may be demonstrable“ (9). Nachdem die Autoren 119 Patientinnen mit Lipödem im Rahmen einer Studie untersucht haben, scheint das Ödem eine noch unbedeutendere Rolle zu spielen. In ihrer 2. Publikation von 1951 schreiben sie: „particularly at the end of the day, there may be some evidence of edema, although the evidence is not great enough to explain the patient's statement relative to the degree of swelling which has occured as a result of orthostatic activity“ (10).

Eine deutlich größere Rolle als das „Ödem“ - dies sei an dieser Stelle nur am Rande vermerkt - spielt in beiden Publikationen die psychische Situation der Lipödempatientinnen. Tatsachlich präsentieren in dieser allerersten Studie zum Lipödem überhaupt nur $24 \%$ der 119 Studienteilnehmer orthostatische Ödeme („minimal to mild pitting edema"), während $29 \%$ an einer "associated Neurosis" litten (11). Auf die Bedeutung dieser psychischen Aspekte wurde bereits ausführlich im ersten und einleitenden Artikel dieser kleinen Reihe zu „Lipödem - Mythen und Fakten“ im "Schwerpunktheft Adipositas“ dieser Zeitschrift eingegangen (12).

Ödeme sind per definitionem Flüssigkeitseinlagerungen.

Klinisch lassen sich diese durch „dellbares“ Weichteilgewebe feststellen. Vielfach haben wir bei unseren Lipödempatientinnen additiv hochauflösende Weichteilsonografien mit einem 18,6-Megahertz-Schallkopf durchgeführt, neuerdings auch mit einem „MoistureMeter" den Grad der Feuchtigkeit im Gewebe gemessen. Mit keiner der genannten Methoden konnten relevante Flüssigkeitseinlagerungen im Gewebe unserer Lipödempatientinnen nachgewiesen werden. Letztlich ist sowohl der klinischinspektorische Befund als auch das sonographische Bild bei der Patientin mit Lipödem als auch bei der Patientin mit einer reinen Lipohypertrophie (schmerzfreie disproportionale Fettgewebsvermehrung) völlig identisch.

- Abbildung 1 zeigt eine Patientin mit Lipödem, Abbildung 2 das sonographische Bild ihrer Oberschenkel (linker und rechter Oberschenkel waren vollkommen identisch). Außer einer Verbreiterung der Subcutis finden sich keine Auffälligkeiten, insbesondere kein Hinweis auf Flüssigkeit.

- Abbildung 3 zeigt eine Patientin mit Lipohypertrophie, Abbildung 4 das sonographische Bild ihrer Oberschenkel 


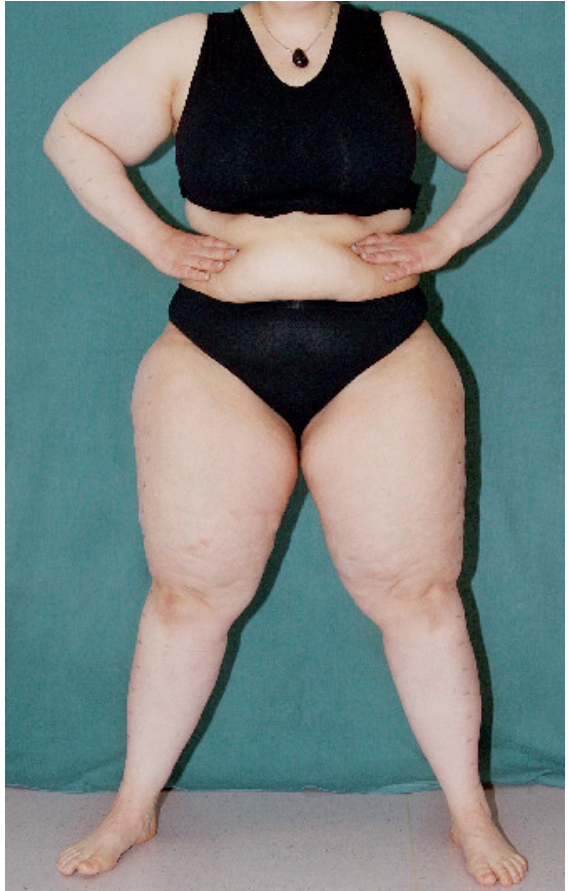

Abb. 1 Patientin mit Lipödem

(auch hier waren linker und rechter Oberschenkel vollkommen identisch). Das klinische wie auch das sonographische Bild ist quasi identisch mit dem der Patientin mit Lipödem.

Die Patientin in $>$ Abbildung 5 leidet an drei klinisch nachweisbaren Krankheiten: An einem distal betonten Lymphödem der Unterschenkel und Vorfüße und einem Lipödem, welches auf die Oberschenkel sowie die proximalen Unterschenkel begrenzt ist. Darüber hinaus liegt eine morbide Adi- positas mit einem BMI von $48 \mathrm{~kg} / \mathrm{m}^{2}$ vor. - Abbildung 6 demonstriert die nachhaltige „Dellbarkeit“ des Lymphödems (unterer Kreis) sowie die "Nichtdellbarkeit" des Weichteilgewebes der Lipödemregion (oberer Kreis).

$\checkmark$ Abbildung 7 und $>$ Abbildung 8 zeigen das sonographische Bild dieser Patientin. In Abbildung 7 (distaler Unterschenkel rechts) präsentiert sich das typische sonographische Bild eines Lymphödems im Stadium 2 mit partieller Aufhebung der Weichteilstrukturen, verdickter Cutis sowie Flüssigkeit im Gewebe (kleine Pfeile). Abbildung 8 (proximaler Oberschenkel) zeigt das typische sonographische Bild eines Lipödems mit unauffälliger Cutis sowie mit Verdickung der Subcutis ohne Hinweis auf Flüssigkeit im Weichteilgewebe.

Allenfalls eine kleine Minderheit unserer Patientinnen mit „reinem“ Lipödem („rein“ im Sinne von Ausschluss anderer Ödemkomponenten wie z.B. kardial oder lymphogen) präsentiert hier im Rahmen unserer klinischen Untersuchung relevante Ödeme - meist in den heißen Sommermonaten.

Führt man den oben begonnenen historischen Blick auf das Lipödem fort, so stellt man fest, dass diese Erkrankung nach den beiden Publikationen der Erstbeschreiber Allen und Hines (1940 und 1951) in eine Art Dornröschenschlaf fiel. In den sechziger und siebziger Jahren gab es allenfalls unsystematische Einzelfallbeschreibungen zum Lipödem bzw. zu Erkrankungen des

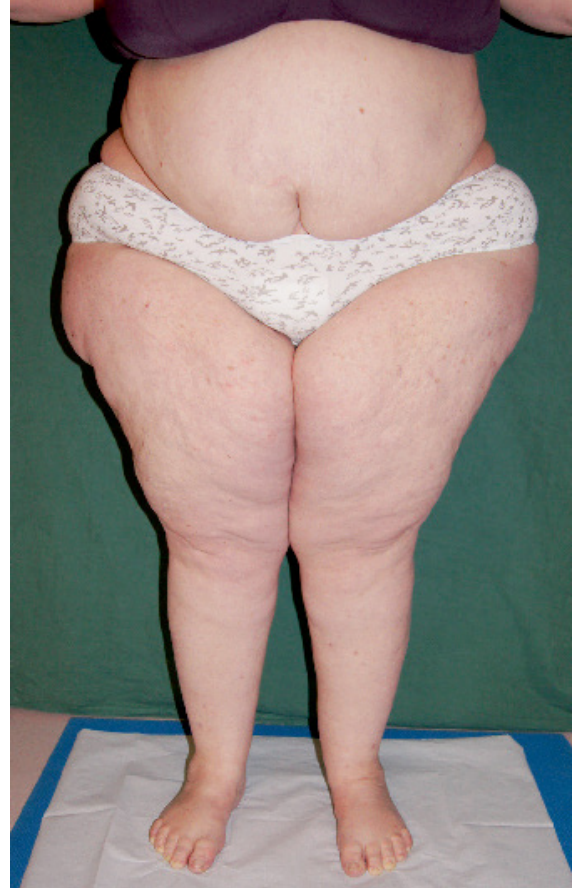

Abb. 3 Patientin mit Lipohypertrophie

schmerzhaften Fettgewebes $(13,14)$. Mit einem Artikel von Schmitz in der Zeitschrift „Gynäkologie“ über das „Lipödem - das dicke Bein der gesunden Frau" kam 1980 wieder Bewegung in dieses Thema (15). Allerdings blieb die Rolle des Ödems Ödem im Sinne von Flüssigkeitseinlagerung - ohne wesentliche Bedeutung! 1982 schrieb Brunner „Pathogenetisch handelt es sich um eine Störung im Verteilungsmuster des subkutanen Fettgewebes" und weiter „Der Fettmantel hat eine sulzige

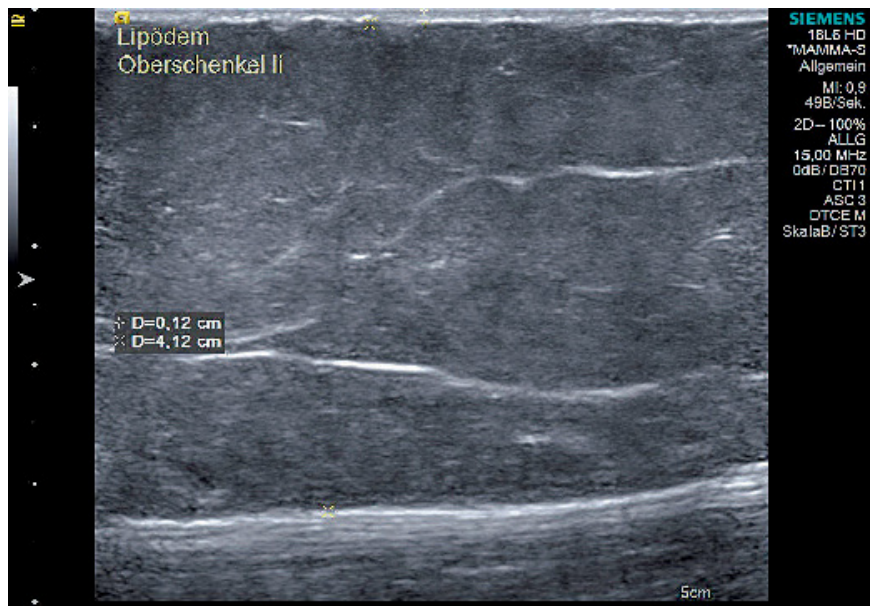

Abb. 2 Sonographisches Bild der Oberschenkel der Patientin mit Lipödem (linker und rechter Oberschenkel waren vollkommen identisch)

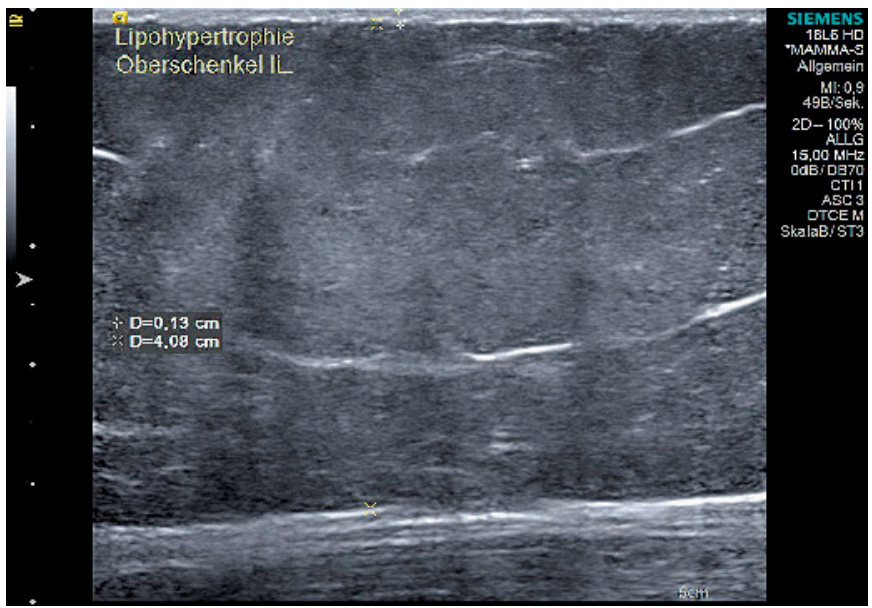

Abb. 4 Sonographisches Bild der Oberschenkel der Patientin mit Lipohypertrophie (auch hier waren linker und rechter Oberschenkel vollkommen identisch) 


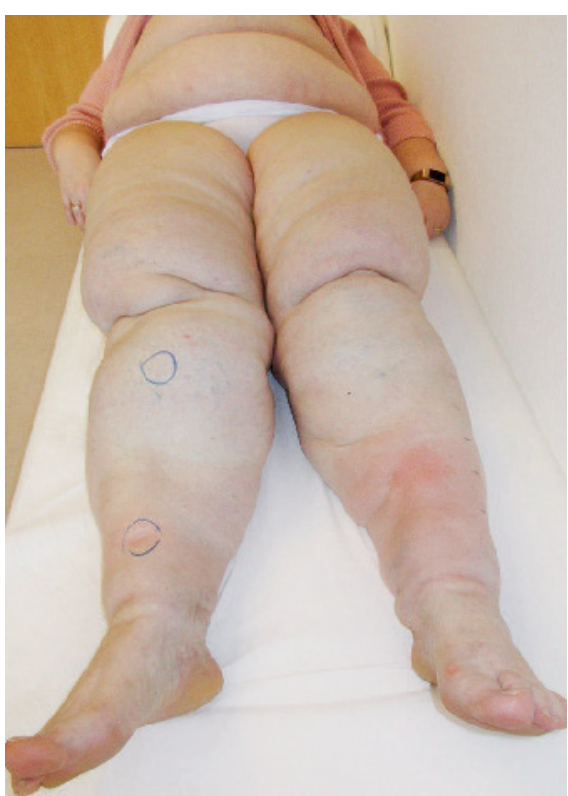

Abb. 5 Patientin mit einem distal betonten Lymphödem der Unterschenkel und Vorfüße und einem Lipödem, welches auf die Oberschenkel sowie die proximalen Unterschenkel begrenzt ist.

Konsistenz und lässt auch über der Tibia auf Druck keine Eindellung zu" (16). Für Gregl (1987) ist das Lipödem ein „mukoides Pseudoödem“. „Im Gegensatz zum kardialen und dystrophischen Ödem bleiben beim Lipödem nach Eindrücken keine Dellen zurück" (17). Rudkin (1994) fand bei seinen Lipödempatientinnen ebenfalls nur "1/4 + edema in the pretibial area" $(1 / 4+$ entspricht einer amerikanischen Ödemklassifikation und bedeutet „kaum dellbar") (18).
Es wird deutlich, dass das „Ödem" im Lipödem eine - wenn überhaupt - eher untergeordnete Rolle spielte.

Nichts desto trotz sehen das Lipödem behandelnde Ärzte dieses kaum bis gar nicht nachweisbare Ödem als Ursache der Beschwerden an. So begründet Cornely seine "lymphologische Liposculptur" (gemeint ist das operative Absaugen von Fettgewebe) mit dem von ihm (und Marsch) entwickelten Konzept der „strukturellen Drainageschwäche“ (19). Nach Cornely „führt das Lipödem zu einer Überproduktion von Lymphflüssigkeit in den Armen und den Beinen, die sich wiederum in einer Druckschmerzhaftigkeit äußert", oder an anderer Stelle, es kommt „zu einer ,Überschwemmung' des Fettgewebes mit Lymphe, die Spannung in den verdickten Extremitäten nimmt zu“ (20).

Dieses Konzept entbehrt jeglicher wissenschaftlichen Evidenz!

Weder ist diese „Überschwemmung des Fettgewebes" klinisch noch sonographisch nachzuweisen.

Auch andere bildgebende Verfahren wie Computertomographie oder Isotopenlymphographie konnten bei Lipödempatientinnen keine Flüssigkeit im Weichteilgewebe oder signifikante Lymphabflussstörungen feststellen (21-23).

Es gibt darüber hinaus auch keine belastbaren histologischen Befunde, die auf ein Ödem im Fettgewebe hindeuten. Kayserling, der Fettgewebe von Lipödempa- tientinnen histologisch untersuchte, stellt fest: „Insgesamt entspricht das Bild einer lokalen Fettgewebsvermehrung im Sinne einer Lipomatose“ und weiter "Zum typischen Bild gehört ein im Wesentlichen unauffälliges Fettgewebe". Auffallend seien lediglich „einzelne herdförmige Fettgewebsnekrosen" sowie vermehrt Makrophagen im Interstitium. „Die Makrophagen exprimieren in typischer Weise Anti-CD68" (24).

Sowohl Fettgewebsnekrosen als auch CD68-positive Makrophagen unterstützen die Hypothese, dass die Schmerzursache beim Lipödem eher auf dem Boden von entzündlichen sowie von hypoxischen Prozessen zu sehen ist. Hierfür sprechen auch laborchemische Untersuchungen an Lipödempatientinnen. Als repräsentative $\mathrm{Pa}$ rameter für oxidativen Stress wurden der Glutathionstatus in den Erythozyten und Malondialdehyd (MDA) im Blutplasma untersucht. MDA lag hier bei Patientinnen mit Lipödem deutlich höher als bei Gesunden und auch die Untersuchung des Glutathions in den Erythrozyten erbrachte ein inhaltlich gleiches Ergebnis (25).

Strößenreuther, der mehrere histologische Untersuchungen des lipödematösen Gewebes im Rahmen seiner Dissertation gesichtet hat, erwähnt in diesem Zusammenhang an keiner Stelle Begriffe, die auf ein „Ödem im Lipödem“ oder auf vermehrte Flüssigkeit im Fettgewebe hindeuten (26).

Aber auch renommierte Lipödemexperten sehen dieses kaum bis meist gar nicht nachweisbare Ödem als Ursache der Beschwerden an. So schreibt z. B. Schmeller 2007: „Durch die Ödeme entstehen Spannungs-, Berührungs- und Druckschmerzen" (27) und in einer späteren Publikation: „The extent of this accumulation, and not the absolute fat volume, is the major reason for sensitivity of the tissue to pressure and touch" (28). Nahezu identisch äußert sich Rapprich, wenn er feststellt, dass die „erhöhte Kapillarpermeabilität, die zu orthostatischen Ödemen führt"..."für die erhöhte Berührungs-und Druckempfindlichkeit des Gewebes verantwortlich" ist (29).

Einmal abgesehen von der Tatsache, dass es für diese Sichtweise weder eine klinische, noch bildgebende noch eine histo- 
logische Evidenz gibt, so drängt sich doch beim Lesen dieser Statements folgende Frage auf: Wenn das Ödem Ursache der Schmerzen der Lipödempatientin sein soll, warum hat dann der Patient mit kardialen Ödemen oder die Patientin mit Lymphödemen keine (oder zumindest keine relevanten) Schmerzen?

Bei diesen Ödemenarten ist Flüssigkeit klinisch als auch sonographisch und histologisch zweifelsfrei darstellbar.

Beim Blick ins europäische Ausland scheinen zumindest die Kollegen in Großbritannien und in den Niederlanden ähnlich kritisch dem „Ödem im Lipödem“ gegenüberzustehen. So schreiben die Autoren der niederländischen Lipödemleitlinien von 2014: „Lipedema is an unfortunate term as it evokes the idea of swelling due to fluid accumulation. However it refers to swelling - in a sense of an increase in volume - due to increased fat tissue" (30). Folgerichtig haben die Autoren der aktuellen niederländischen Leitlinien das Symptom „Ödem“ in ihrem Kriterienkatalog, der das Lipödem definiert, erst gar nicht aufgeführt.

Dass das „Ödem“ nichts mit Ödem - im Sinne von Flüssigkeit - zu tun hat, spiegelt sich auch in den aktuellen britischen „lipedema guidelines" von 2017 wider: "the word lipoedema means, fat swelling “" (31).

Warum ist die Frage nach dem Ödem so wichtig, warum hat die Klärung dieses Statements eine so immense praktische Bedeutung?

Wie oben aufgeführt, wird in einer Vielzahl von nationalen wie auch internationalen Publikationen die Manuelle Lymphdrainage als regelmäßig durchzuführende Standardtherapie des Lipödems betrachtet. Hintergrund für diese Betrachtung ist die Auffassung, dass das Ödem für die Schmerzsymptomatik verantwortlich sei. Wenn aber nun gar kein Ödem (oder zumindest kein relevantes) bei Lipödempatientinnen nachweisbar ist, auf welcher Grundlage erfolgt dann die Verordnung von Manueller Lymphdrainage?

Für die Verordnung von Manueller Lymphdrainage beim Lipödem sollten zwei Fragen zweifelsfrei geklärt werden:

1. Gibt es eine wissenschaftliche Evidenz dafür, dass dieses kaum bis gar nicht

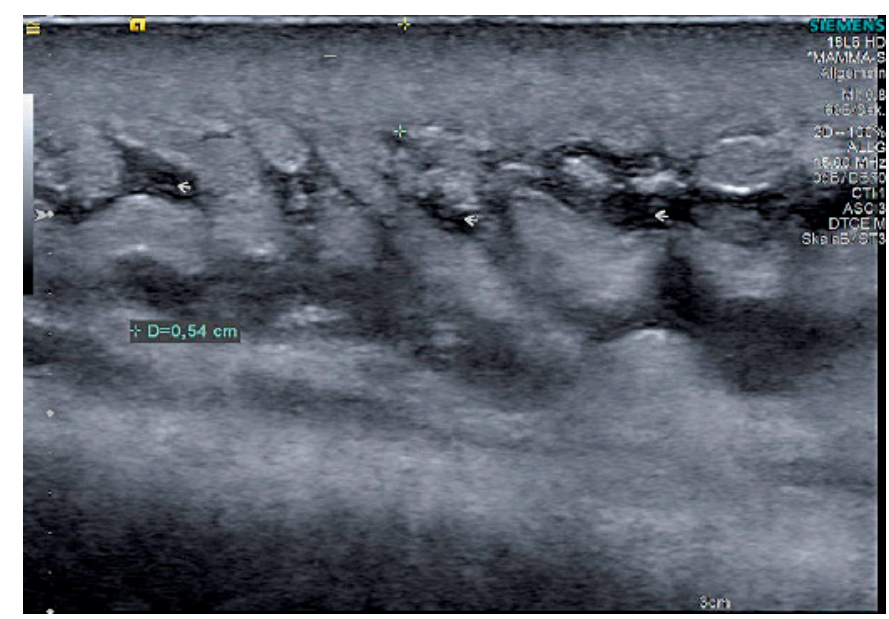

Abb. 7

Distaler Unterschenkel rechts der Patientin aus Abbildungen 5 und 6: Es präsentiert sich das typische sonographische Bild eines Lymphödems im Stadium 2 mit partieller Aufhebung der Weichteilstrukturen, verdickter Cutis sowie Flüssigkeit im Gewebe (kleine Pfeile).

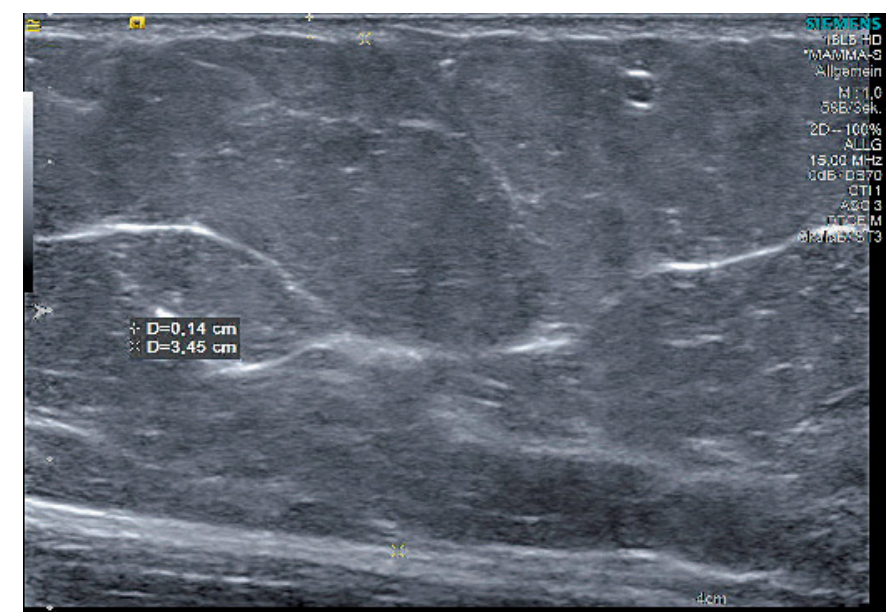

Abb. 8

Proximaler Oberschenkel der Patientin aus Abbildungen 5 und 6 : Es zeigt sich das typische sonographische Bild eines Lipödems mit unauffälliger Cutis sowie mit Verdickung der Subcutis ohne Hinweis auf Flüssigkeit im Weichteilgewebe

vorhandene Ödem für die Beschwerden der Patientinnen verantwortlich ist?

2. Gibt es eine wissenschaftliche Evidenz dafür, dass Manuelle Lymphdrainage durch ihre Drainageeffekte die Beschwerden unserer Lipödempatientinnen verbessert?

Beide Fragen müssen nach jetzigem Kenntnisstand explizit verneint werden!

In unserer kassenärztlichen lymphologischen Ambulanz sind wir täglich vielfach mit der dringenden Forderung von Lipödempatientinnen nach Manueller Lymphdrainage konfrontiert. Häufig werden uns Lipödempatientinnen zugewiesen, denen seit vielen Jahren zweimal (manchmal auch dreimal) wöchentlich Manuelle Lymphdrainage verordnet wird. Es muss betont werden, dass dies medizinischer, aber auch ökonomischer Unsinn ist! Viele Lipödempatientinnen weisen - intensiv - darauf hin, dass ihnen „die Lymphdrainage gut tut". Aber - und auch das sollte betont werden - "gut tun“ ist nicht dasselbe wie medizinisch notwendig.

Möglicherweise sind es ganz andere Effekte der Manuellen Lymphdrainage, die von den Patientinnen als angenehm erlebt werden. Effekte, die nur wenig mit Entstauung zu tun haben.

Die große Mehrheit unserer Lipödempatientinnen präsentiert behandlungswürdige psychische Symptome; psychische Symptome, die ganz unabhängig vom Lipödem aufgetreten sind. Viele unserer Patientinnen mit Lipödem leiden darüber hinaus auch an depressiven Störungen, Angst - oder Essstörungen. Dies wird auch in einer Pilotstudie unserer Klinik bestätigt, die bereits im Einführungsartikel dieser kleinen Reihe zum Lipödem vorgestellt wurde (32). 
Ausgehend von den Ergebnissen unserer Pilotstudie vermuten wir daher andere Aspekte innerhalb der Manuellen Lymphdrainage, die von den Patienten als positiv und angenehm wahrgenommen werden: Aspekte, die mehr den Massageeffekt betonen, Aspekte, die mehr mit der Reduktion von Stress und Erschöpfung zu tun haben als mit Entstauung. Die Datenlage zum Einfluss von Massagetherapie auf psychische Symptome wie Angst und Depression ist konsistent $(33,34,35)$. Darüber hinaus kann davon ausgegangen werden, dass die Wertschätzung der Manuellen Lymphdrainage seitens der Patientinnen vor allem auch in der Berührung und der menschlichen Zuwendung, im Kontakt zwischen Therapeut (bzw. Therapeutin) und Lipödempatientin liegt. Nicht zuletzt wird durch den Akt der Therapie auch die Krankheit Lipödem anerkannt. Patienten mit Lipödem haben häufig eine Odyssee hinter sich gebracht, bis die Beschwerden endlich ernst genommen, das Lipödem diagnostiziert und anbehandelt wurde.

Psychische, psychosoziale und gesellschaftliche Faktoren haben erheblichen Einfluss auf die Beschwerden unserer Lipödempatientinnen. Dennoch - und das sei betont - ist das Lipödem primär eine somatische Erkrankung.

Das Lipödem entwickelt sich immer aus einer Lipohypertrophie heraus, allerdings nur bei einem sehr kleinen Teil der Betroffenen. Warum sich bei manchen Patientinnen (sehr selten sind auch Männer betroffen) eine Schmerzsymptomatik im Fettgewebe entwickelt und welche Pathophysiologie dieser Beschwerdesymptomatik zugrunde liegt, ist zum jetzigen Zeitpunkt noch unklar und Gegenstand von Spekulationen.

Neben der oben dargestellten „Ödemhypothese" kursieren weitere divergierende Vermutungen hinsichtlich der Ursachen des Schmerzes durch die wissenschaftliche Literatur. Brenner hat diese in einer Übersichtsarbeit weitgehend umfassend zusammengestellt (36).

Konsens besteht allenfalls in der Frage, dass der Ort des Schmerzgeschehens im subkutanen Fettgewebe der Extremitäten liegt.

Da dieser Schmerz im subkutanen Fettgewebe üblicherweise zum Zeitpunkt der
Diagnosestellung bereits länger als 6 Monate andauert, kann er als chronisch bezeichnet werden.

Ursachen für chronische Schmerzen sind i.d.R. degenerative Prozesse des Bewegungsapparates (z.B. Arthrose), Schmerzen durch Ischämie (z.B. periphere arterielle Verschlusskrankheit), neuropathische Schmerzen (z.B. nach Apoplex, diabetische Polyneuropathie), Tumorschmerzen sowie entzündliche Schmerzen (z. B. bei Rheumatoider Arthritis).

Beim Blick auf die Literatur zum expandierenden subkutanen Fettgewebe werden im Wesentlichen zwei Vorgänge genannt: die Inflammation des Fettgewebes und die Hypoxie. So schreiben z. B. Pou et al. zum subkutanen Fettgewebe: „appear to be associated with chronic inflammation" (37) und Rutkowky et al stellen fest: „Adipose expansion results in tissue hypoxia" (38). Auch die oben bereits genannten Befunde von Kayserling weisen in diese Richtung. Ganz aktuell sind die Arbeiten von Crescenzi et al., die Fettgewebe von Lipödempatientinnen sowie einer vergleichbaren Gruppe ohne Lipödem untersuchten. Den Autoren dieser Studie fiel eine Erhöhung des Natriumgehalts der Haut bei Lipödempatientinnen auf (,skin sodium is elevated in women with lipedema") und weiter: "Skin sodium accumalation is an ermerging hallmark of inflammatory diseases" (39).

Entzündung im Sinne eines „chronic low grade state of inflammation" (40) und Hypoxie bieten das bislang plausibelste Erklärungsmodell für die Schmerzen unserer Lipödempatientinnen. Therapiekonzepte sollten daher in jedem Falle auch auf diese mögliche Schmerzursache fokussieren.

\section{Fazit}

Es gibt keine Evidenz dafür, dass ein Ödem bei der Erkrankung Lipödem eine relevante Rolle spielt, noch dass es für die Beschwerden der Patientinnen verantwortlich ist. Manuelle Lymphdrainagen sollten daher auch nur seriell und bei klinischem Nachweis von Ödemen verordnet werden. Dies wäre z. B. in den heißen Sommermonaten und entsprechendem Leidensdruck durch das orthostatische Ödem möglich.
Für die regelmäßig wöchentliche Durchführung von Manuellen Lymphdrainagen beim Lipödem gibt es keinerlei Grundlage!

Wie eingangs festgestellt, ist das Lipödem weit mehr als nur "dickere, schmerzhafte Beine“.

Darum müssen wir manche der alten Pfade verlassen, Pfade, für die es keine wissenschaftliche Evidenz gibt, Pfade, die darüber hinaus auch unserer klinischen Erfahrung widersprechen.

Eine umfassende Therapie des Lipödems sollte daher auch all jene Aspekte berücksichtigen, die nicht so offensichtlich sind wie das Augenscheinliche und das vordergründig Geäußerte. Lipödem-Therapie muss neben der Behandlung der somatischen Beschwerden auch auf die bereits beschriebenen Aspekte dieses komplexen Krankheitsbildes fokussieren, nämlich auf:

- seriöse Aufklärung

- Behandlung des Schmerzes

- die psychische Vulnerabilität unserer Lipödempatientinnen

- die Gewichtsprogredienz

- Selbstakzeptanz in Zeiten des „Magermodelwahns"

Die Vorstellung eines umfassenden Therapiekonzeptes für Lipödempatientinnen wird Inhalt im letzten Teil unserer kleinen Lipödemreihe sein.

Neue Wege entstehen, in dem wir sie gehen - dies gilt auch für die Therapie des Lipödems!

\section{Interessenkonflikt}

Nach Angaben der Autoren bestehen keine Interessenkonflikte.

\section{Ethische Richtlinien}

Für das Manuskript wurden keine Studien an Menschen oder Tieren durchgeführt.

\section{Literatur}

1. Bertsch T, Erbacher, G. Lipödem - Mythen und Fakten Teil 1. Phlebologie 2018; 47: 84-92.

2. Herbst KL. Rare adipose disorders (RADs) masquerading as obesity. Acta Pharmacol Sin 2012 Feb; 33(2): 155-172.

3. Buck W, Herbst KL. Lipedema: A Relatively Common Disease with Extremely Common Miscon- 
ceptions. Plast Reconstr Surg Glob Open 2016 Sep; $4(9)$.

4. S 1 Leitlinie Lipödem S. 11. Abrufbar unter http://www.awmf.org/uploads/tx_szleitli nien/037-0121_S1_Lipoedem_2016-01.pdf

5. Schmeller W, Meier-Vollrath I. Lipödem in Weissleder $\mathrm{H}$ Schuchhardt $\mathrm{CH}$ (Hrsg). Erkrankungen des Lymphgefäßsystems, 5 Auflage. Köln: Viavitalverlag 2011, S. 400.

6. Herpertz U. Ödeme und Lymphdrainage, 3. Auflage. Stuttgart: Schattauer 2006, S. 181.

7. Verein zur Förderung der Lymphödemtherapie e.V. Abrufbar unter http://www.lymphverein.de/ verordnung_von_mld.html

8. Meier-Vollrath I et al. Lipödem: Verbesserte Lebensqualität durch Therapiekombination. Dtsch Arztebl 2005; 102(15): A-1061.

9. Allen E, Hines E. Lipedema of the legs: a syndrome characterized by fat legs and orthostatic edema. Proc Staff Mayo Clin 1940; 15: 184-187.

10. Wold LE Hines EA Allen EV Lipedema of he legs; a syndrome characterized by fat legs and edema. Ann Intern Med. 1951; 34(5): 1243-1250.

11. Wold LE Hines EA Allen EV: a.a.O. 1248-1249.

12. Bertsch T, Erbacher, G. Lipödem - Mythen und Fakten Teil 1. Phlebologie 2018; 47: 84-92.

13. Greer KE. Lipedema of the legs. Cutis 1974; 14 98-100.

14. Müller W. Die Pannikulose. Z. Rheumaforschung 1973; 32: 169-176

15. Schmitz R. Das Lipödem. Gynäkologe 1980; 13: 102-105.

16. Brunner U. Vaskuläre Erkrankungen bei Lipödem der Beine. Schweiz Med Wschr 1982; 112, Nr. 33, 1131.

17. Gregl A. Das Lipödem. Lymphologie 1987; XI, 41-3, 5-6.

18. Rudkin GH, Miller TA. Lipedema: A Clinical Entity Distinct from Lymphedema. Plast Reconstr. Surg. 1994 Nov; 94 (6) 842
19. Cornely M. Das Lipödem an Armen und Beinen. Teil 1: Pathophysiologie. Phlebologie 1/2011 23.

20. Cornely M. Das Lipödem an Armen und Beinen. Teil 2: Zur konservativen und operativen Therapie des Lipödems, genannt Lipohyperplasia dolorosa. Phlebologie 3/2011 146

21. Kappos EA. Lipedema: Diagnostic and management challenges International J of Women's Health Volume 8 (Issue 1) 2016; 389-395.

22. Monnin-Delhom ED et al. High resolution unen hanced computed tomography in patients with swollen legs. Lymphology 2002; 35(3): 121-128.

23. Tietjen KU, Schulz-Ehrenburg U. Isotopenlymphographische Befunde beim Lipödem. In: Holzmann H., Altmeyer P., Hör G., Hahn K. (eds) Dermatologie und Nuklearmedizin. Berlin, Heidelberg: Springer 1985 .

24. Kayserling E. Zur Histologie des Lipödems. In Strößenreuther RHK. Lipödem und Cellulitis sowie andere Erkrankungen des Fettgewebes. Köln: Viavital Verlag 2001.

25. Brenke R, Siems WG. Hinweise für die Beteiligung freier Radikaler an der Pathogenese des Lipödems. In Strößenreuther RHK. Lipödem und Cellulitis sowie andere Erkrankungen des Fettgewebes. Köln: Viavital Verlag 2001.

26. Strößenreuther RHK. Das Lipödem. Neue Aspekte der Pathophysiologie, Diagnostik und Therapie sowie weiterführende differentialdiagnostische Betrachtungen. Unveröffentlichte Dissertation. Technische Universität München 1999.

27. Schmeller W, Meier-Vollrath I. Das Lipödem: Neue Möglichkeiten der Therapie. Schweiz Med Forum 2007; 7: 151

28. Schmeller W, Meier-Vollrath I. Lipedema. Abrufbar unter https://www.lipedema-simplified.org/ downloads/Lipoedema-schmeller.pdf S. 297

29. Rapprich $\mathrm{S}$ et al. Liposuction is an effective treatment for lipedema - results of a study with $25 \mathrm{pa}-$ tients. Journal of the German Society of Dermatology $2011 ; 1(9)$
30. Lipedema - Guidelines in the Netherlands 2014. Abrufbar unter https://diseasetheycallfat.tv/wpcontent/uploads/2015/08/Dutch-lipoedemaguideline-2014.pdf

31. Wounds UK. Best Practice Guidelines The management of lipoedema. 2017. Abrufbar unter http://www.wounds-uk.com/pdf/con tent_11941.pdf

32. Bertsch T, Erbacher, G. Lipödem - Mythen und Fakten Teil 1. Phlebologie 2018; 47: 84-92.

33. Rauchfuss $M$ et al. Massagetherapie reduziert Schmerzen, Erschöpfung und Stress bei Mammakarzinompatientinnen. Geburtsh Frauenhk 2010; 70: 870-824.

34. Baumgart $S$ et al. Wirksamkeit der Massagetherapie bei Depression und Angsterkrankungen sowie bei Depressivität und Angst als Komorbidität - Eine systematische Übersicht kontrollierter Studien. Phys Med Rehab Kuror 2011; 21: 167-182

35. Bita Ajilchil $\mathrm{H}$ et al. The Effectiveness of Massage Therapy on Reducing Depression in Students. Indian Journal of Fundamental and Applied Life Sciences. 2015; Vol. 5 (S3) 1937-1942.

36. Brenner E. Wie kommt der Schmerz ins Lipödem. LymphForsch 21 (1) 2017 40-47.

37. Pou KM et al. Visceral and Subcutaneous Adipose Tissue Volumes Are Cross-Sectionally Related to Markers of Inflammation and Oxidative Stress. The Framingham Heart Study. Circulation published online Aug 2007; 20.

38. Ruktowki J et al. Mechanisms of Obesity and Related Pathologies: The Macro- and Microcirculation of Adipose Tissue. FEBS Journal. 2009 October 2009; 276 (20) 5738-5746.

39. Crescenzi R et al. Tissue Sodium Content is Elevated in the Skin and Subcutaneous Adipose Tissue in Women with Lipedema. Obesity 2018; 26 (2): 316.

40. Mancuso P. The role of adipokines in chronic inflammation. Immotargets Ther 2016; 5: 47-56. 